\title{
Pain in the RIG?
}

\author{
S. Biswas, D. Bromley and Y. M. Miao \\ Ipswich Hospital NHS Trust, Heath Road, Ipswich, Suffolk IP4 5PD,UK
}

Gastrostomy tubes can be inserted either endoscopically or radiologically, depending on practicality of insertion and clinical need ${ }^{(1-3)}$. This study aims to compare complications of PEG and RIG procedures and also to compare pain experienced post-procedure. The standard is for the patient to be pain-free post insertion. We looked at whether the presence of pain and significant complications may affect the decision as to which type of gastrostomy should be used.

A retrospective 3-year study in 2004 at Ipswich Hospital NHS Trust highlighted that following 50 PEG insertions there were three complications, two minor and one major (a mesenteric bleed requiring laparotomy). Our 3-year retrospective study in 2009 highlighted that following 37 RIG insertions (78\% inserted for head and neck cancers, $22 \%$ for stroke, other reasons included motor neurone disease and cerebral palsy), there were 13 complications of which two were major (both laparotomies for leak of gastric contents). This indicates a major complication rate of $2 \%$ in PEGs compared to $5.4 \%$ in RIGs. Minor complications included wound infection, gastrostomy stoma site leak, tube displacement or blockage and post-procedure pain. No 30-day mortality occurred following either procedure.

A six-month prospective audit was carried out to compare pain perception with the use of a questionnaire $4 \mathrm{~h}$ post-procedure and on the following day. There were 13 PEG insertions (76\% stroke patients, others were for motor neurone disease, head injury and head and neck cancer) and seven RIG insertions (all for head and neck cancers). A larger proportion of RIG patients reported pain both at $4 \mathrm{~h}$ ( $43 \%$ compared to $38 \%$ of PEG patients) and the following day (14\% compared to $8 \%$ of PEG patients), and these patients were noted to require more analgesia. This may have been due to the type of local anaesthetic used, need for tract dilatation during RIG insertion and the use of suture anchors.

At Ipswich Hospital, RIGs insertions carry a higher complication rate and cause more pain when compared to PEGs. RIGs are mostly used for patients with head and neck cancers which may carry a risk of tumour seeding if an endoscopic approach is undertaken. In conclusion, PEGs are the preferred route for enteral feeding in the majority of patients unless contra-indicated by clinical need.

1. Laasch HU, Wilbraham L, Bullen K, et al. (2003) Clin Radiol 58, 398-405.

2. Silas AM, Pearce LF, Lestina LS, et al. (2005) Eur J Radiol 56, 84-90.

3. Rustom IK, Jebreel A, Tayyab M, England RJ \& Stafford ND (2006) J Laryngol Otol 120, 463-466. 